\title{
Die Bremer Bürgerschaftswahl vom 13. Mai 2007: Neustart für Rot-Grün und Fünf- statt Dreiparteiensystem
}

\author{
Beate Hoecker
}

\section{Die Ausgangslage}

Die Bremer Bürgerschaftswahl vom 13. Mai 2007 wurde mit Spannung erwartet, denn zum einen war sie die einzige Landtagswahl des Jahres 2007, zum anderen zeigte die seit zwölf Jahren regierende Große Koalition in Bremen deutliche Erosionserscheinungen. Ihr Hauptziel, nämlich die Sanierung oder zumindest die Konsolidierung des Haushalts, hatte sie auch in der dritten gemeinsamen Wahlperiode weit verfehlt. Stattdessen erreichten Neuverschuldung und Gesamtdefizit - trotz Sonderzuschüssen aus Bundestöpfen - ein Rekordniveau; annähernd zwei Milliarden Euro fehlten im Doppelhaushalt für 2006/07¹.

Mitverantwortlich für diese extreme Haushaltsnotlage waren unter anderem wirtschaftspolitische Fehlentscheidungen. So blieb ein überdimensioniertes Investitionsprogramm weit hinter den Erwartungen zurück, und neben dem Musical-Haus erwies sich auch der mit einem dreistelligen Millionenbetrag geförderte Space Park als Flop und musste kaum ein Jahr nach seiner Eröffnung wieder schließen ${ }^{2}$. Daneben verharrte die Arbeitslosigkeit nahezu unverändert auf Rekordniveau. Hinzu kamen innerhalb einiger Ressorts Affären beziehungsweise Vorfälle, die unter anderem zu Rücktritten von Senatsmitgliedern führten und das Vertrauen der Bremer Wählerschaft in die Kompetenzen der politisch Verantwortlichen zweifellos minderten ${ }^{3}$.

Diese politische Stimmung schlug sich auch in den Umfragen vor der Wahl nieder. Die Zufriedenheit mit der Senatsarbeit von Rot-Schwarz ging im Vergleich zu 2003 weiter zurück, wobei die SPD allerdings noch erkennbar besser abschnitt als die CDU4. Eine Neuauflage der Großen Koalition wünschten folglich nur noch 37 Prozent der Befragten (2003: 45 Prozent), während 23 Prozent für die Alternative Rot-Grün votierten (2003: 21 Prozent) 5 .

1 Vgl. Forschungsgruppe Wahlen e.V., Wahl in Bremen. Eine Analyse der Bürgerschaftswahl vom 13. Mai 2007, Bericht Nr. 128, Mannheim 2007, S. 16 (Online-Dokument).

2 Vgl. Lothar Probst, Große Koalitionen als Sanierungsmodell? Erfahrungen aus Bremen, in: ZParl, 37. Jg. (2006), H. 3, S. $626 \mathrm{ff}$.

3 So musste der CDU-Wirtschaftssenator Peter Gloystein im Mai 2005 zurücktreten, nachdem er bei der Eröffnung eines Weinfestes einem Obdachlosen von der Bühne herunter Sekt über den Kopf gegossen hatte. Vgl. Sektattacke kostet Regierungsvize Gloystein das Amt, in: sueddeutsche. de vom 13. Mai 2005 (www.sueddeutsche.de/deutschland/artikel/92/53039/article.html). Auch Sozialsenatorin Karin Röpke gab im Oktober 2006 ihr Amt zurück und übernahm damit die Verantwortung für schwere Versäumnisse ihrer Behörde im Fall „Kevin“. Vgl. Leichenfund zwingt Senatorin zum Rücktritt, in: Spiegel Online vom 11. Oktober 2006 (www.spiegel.de/panorama/ justiz/0,1518,druck-441998,00.html). Daneben erregte der so genannte Klinik-Skandal, zu dessen Aufklärung die Bürgerschaft einen Untersuchungsausschuss einsetzte, große öffentliche Aufmerksamkeit.

4 Auf einer Skala von +5 bis -5 erreichte die Koalition jetzt 0,8 $(2003: 1,1)$, die SPD bekam die Note 1,1 (2003: 1,0), die CDU 0,6 (2003: 1,0). Vgl. Forschungsgruppe Wahlen e.V., a.a.O., S. 24.

5 Sonstige Varianten nannten 20 Prozent, und 20 Prozent wussten keine Antwort. Vgl. ebenda, S. $24 \mathrm{f}$. Ein anderes Bild ergibt sich allerdings, wenn nur Rot-Schwarz oder Rot-Grün zur Alter- 


\section{Der Wahlkampf}

Moderne Wahlkämpfe zeichnen sich bekanntlich dadurch aus, dass sie als Entscheidungen zwischen personellen Alternativen stilisiert werden. Dies gilt auch für die Bremer Bürgerschaftswahl, bei der SPD wie CDU zudem jeweils mit neuen Spitzenkandidaten im Vergleich zur Wahl 2003 antraten.

Für die Sozialdemokraten stellte der personelle Übergang von Henning Scherf zu Jens Böhrnsen eine besondere Herausforderung dar, denn wie kein anderer Bremer Politiker seit der Ära Hans Koschnick war Scherf als Landesvater überaus populär und hatte bei den vergangenen Wahlen wichtige Punkte für die SPD holen können. Jens Böhrnsen, seit November 2005 bereits Bürgermeister und Präsident des Senats, verfolgte daher die Strategie eines bürgernahen Präsenzwahlkampfes, um seinen Bekanntheitsgrad und seine Popularität zu erhöhen. Bereits Wochen vor der Wahl absolvierte der ehemalige Verwaltungsrichter und Ex-Fraktionschef zahlreiche Termine in den Stadtteilen, bei Vereinen, Verbänden und Initiativen. Zudem wurde die Imagebroschüre „Jens Böhrnsen - Der Neue“ verteilt und eine Kandidatenhomepage ins Internet gestellt, die neben biographischen Angaben auch Reden und Regierungserklärungen von Böhrnsen sowie das SPD-Wahlprogramm „Gemeinsam Zukunft gestalten" enthielt ${ }^{6}$.

In diesem Wahlprogramm, das als „Regierungsprogramm 2007-2011“7 vorgelegt wurde und dessen gedruckte Version vom Layout eher den spröden Charme eines kommentierten Vorlesungsverzeichnisses ausstrahlte, zog die SPD eine weitgehend positive Leistungsbilanz ihrer Regierungsarbeit und deckte alle relevanten Themenfelder des Stadtstaates ab; eine besondere Schwerpunktsetzung war allerdings nicht zu erkennen. Erst im Wahlkampf selbst stellte sie Arbeit, Bildung und sozialen Zusammenhalt in den Vordergrund ${ }^{8}$ und entschied sich damit für klassisch sozialdemokratische Politikbereiche, in denen sie zudem eine besondere Problemlösungskompetenz besaß. So lag die SPD in den Politikfeldern Bildung (SPD: 34 / CDU: 19 Prozent), Familie (SPD: 35 / CDU: 18 Prozent) und Soziales (SPD: 36 / CDU: 19 Prozent) deutlich vor der CDU; beim Thema Arbeitsmarkt dagegen wurde den beiden großen Parteien eine annähernd gleiche Lösungskompetenz zugeschrieben (SPD: 26 / CDU: 27 Prozent) $)^{9}$.

Auf Tradition setzte die SPD auch hinsichtlich ihrer Plakate, die in einem tiefen Rot gehalten waren und Bremen ein gutes Zeugnis ausstellten: „Bremen begeistert“, „Bremen wächst“, „Bremen fördert“, so lauteten unter anderem die Slogans. Stand anfangs allein die Botschaft auf den Großflächenplakaten, war es zum Schluss allein der Spitzenkandidat, der „Echt Bremen“ verkörpern sollte ${ }^{10}$. Im Unterschied zu seinem Vorgänger Henning Scherf setzte Böhrnsen jedoch nicht auf eine Fortführung der Großen Koalition ${ }^{11}$, sondern hielt

native stehen. 59 Prozent würden sich dann für das bisherige Regierungsbündnis entscheiden und 36 Prozent für Rot-Grün. Vgl. ebenda, S. 25.

6 Vgl. www.jens-boehrnsen.de.

7 Vgl. Gemeinsam Zukunft gestalten. Regierungsprogramm 2007-2011, beschlossen auf dem Landesparteitag der SPD am 17. Februar 2007.

8 „Wenn Sie mich wählen, werde ich mich weiter einsetzen für mehr Arbeit, bessere Bildung und sozialen Zusammenhalt“, so Jens Böhrnsen im Weser-Report vom 9. Mai 2007, S. 3.

9 Vgl. Forschungsgruppe Wahlen e.V., a.a.O., S. 33, S. 35.

10 Vgl. hierzu: Plakate, die Politik versprechen, in: Weser Kurier (WK) vom 14. April 2007, S. 13.

11 Scherf hatte 2003 eine rot-grüne Koalition ausgeschlossen, da das achtjährige Bündnis mit der CDU sehr erfolgreich gewesen sei. Vgl. hierzu Reinhold Roth, Die Bremer Bürgerschaftswahl vom 
die Koalitionsfrage offen: „Ich gehe in die Bürgerschaftswahl nicht als Koalitionär. Ich bin Sozialdemokrat, also kein Rot-Schwarzer und auch kein Rot-Grüner. Ich bin ein Roter."12

Für die Bremer CDU stellte die fehlende Koalitionsaussage seitens der SPD zweifellos ein Handikap dar, denn angesichts ihrer mageren Umfragewerte hatten sich die Christdemokraten 2007 erneut für einen Koalitionswahlkampf entschieden, um weiter mitregieren zu können. Da sie die SPD als politischen Gegner folglich nicht direkt angreifen konnten, beschränkten sie sich darauf, vor rot-grünen Experimenten zu warnen ${ }^{13}$ und besetzten stattdessen soziale Themen, um der SPD auf deren ureigenen Feldern Konkurrenz zu machen. In ihrem Regierungsprogramm versprach die CDU „eine neue Agenda für die Bremer Politik" 14 und richtete den Fokus insbesondere auf die Bereiche Bildung, Familie, Soziales und Wertevermittlung. Da der CDU in diesen Politikfeldern jedoch durchweg eine geringere Problemlösungskompetenz zugeschrieben wurde als der SPD, stellte diese inhaltliche Festlegung ein gewagtes Unternehmen dar. Nur eine geringe Rolle im Wahlkampf spielten demgegenüber die Themen mit einem klaren Kompetenzvorsprung der CDU, nämlich Finanzen (CDU: 29 / SPD: 21 Prozent) und Kriminalität (CDU: 31 / SPD: 17 Prozent) ${ }^{15}$.

Daneben betonte die CDU ihren „maßgeblichen Anteil daran, dass die Große Koalition für das Land Bremen ein Erfolgsmodell“16 sei. Eine „kluge Wirtschaftspolitik“"17, neue Arbeits- und Ausbildungsplätze, attraktive Wohnangebote und die Wiederherstellung von Sicherheit und Ordnung waren demnach in erster Linie ihrer Regierungsbeteiligung zu verdanken ${ }^{18}$. Diese positive Selbstdarstellung deckte sich allerdings nur zum Teil mit der Beurteilung innerhalb der Wählerschaft. Gerade bei den für Bremen wichtigen Feldern Wirtschaft und Arbeitsplätze hatte die CDU ihren klaren Kompetenzvorsprung von 2003 verloren und lag jetzt etwa gleichauf mit der SPD ${ }^{19}$.

Der neue Spitzenkandidat der Bremer CDU, der Innensenator und seit 2005 auch Bürgermeister Thomas Röwekamp, bemühte sich nach Kräften, die positive Leistungsbilanz sowie die an sozialen Themen ausgerichtete Wahlkampfstrategie in der Öffentlichkeit glaubhaft zu vermitteln. Neben den üblichen Wahlkampfauftritten war er zu diesem Zweck häufig in seinem "Café Röwekämp“20 als Ansprechpartner für die Bevölkerung präsent.

25. Mai 2003: Triumph für Henning Scherf und Bestätigung der Großen Koalition, in: ZParl, 35. Jg. (2004), H. 2, S. 243.

12 So Böhrnsen im Gespräch, in: Echt Bremen. Das Magazin der SPD Bremen zur Wahl 2007, S. 12.

13 „Wenn Sie mich wählen, bekommt Bremen eine starke Regierung statt rot-grüner Experimente“, so der CDU-Spitzenkandidat Thomas Röwekamp im Weser-Report vom 9. Mai 2007, S. 3. Auch auf Plakaten der CDU war der Slogan „Starkes Bremen statt Rot-Grün“ zu lesen.

14 Regierungsprogramm der CDU-Bremen für die 17. Legislaturperiode der Bremischen Bürgerschaft, beschlossen auf dem Landesparteitag der CDU Bremen am 9. März 2007, S. 5 (OnlineDokument http://www.cdu-bremen.de).

15 Vgl. Forschungsgruppe Wahlen e.V., a.a.O., S. 33, S. 35.

16 Regierungsprogramm der CDU-Bremen, a.a.O., S. 4.

17 Ebenda.

18 Vgl. ebenda.

19 Bei „Schaffung von Arbeitsplätzen“ kam die CDU auf 27, die SPD auf 26 Prozent; bei „Wirtschaftsfragen" erhielt die CDU 24, die SPD 26 Prozent. Vgl. Forschungsgruppe Wahlen e.V., a.a.O., S. 33.

20 In guter Innenstadtlage hatte Röwekamp ein ehemaliges Ladenlokal zum Wahlkampflokal umrüsten lassen und bot dort Kaffee und politische Informationen an. Der Name „Röwekämp“ sollte in Anlehnung an den Begriff „Camp“ an die Ruderregatta vom August 2006 sowie an den Spitzenkandidaten erinnern. 
Gleichzeitig gab es auch von ihm eine Kandidatenhomepage im Netz ${ }^{21}$. Doch trotz aller Anstrengungen blieb der Rechtsanwalt ein eher blasser Spitzenkandidat, der zudem im direkten Vergleich mit dem siebzehn Jahre älteren Böhrnsen deutlich unterlegen war. Nach einer Vorwahlumfrage der Forschungsgruppe Wahlen wünschten Anfang Mai lediglich 19 Prozent Röwekamp als Regierungschef, während sich 56 Prozent für Böhrnsen aussprachen ${ }^{22}$. Auch hinsichtlich persönlicher Eigenschaften schnitt Röwekamp in der Einschätzung der Wählerschaft durchweg schlechter ab als sein Gegner von der SPD (vgl. Tabelle 1).

\begin{tabular}{|c|c|c|c|}
\hline & Böhrnsen & Röwekamp & beide gleich \\
\hline glaubwürdiger & 25 & 4 & 48 \\
\hline tatkräftiger & 21 & 13 & 40 \\
\hline sympathischer & 45 & 9 & 23 \\
\hline eher bürgernah & 34 & 10 & 31 \\
\hline eher ein Siegertyp & 24 & 19 & 31 \\
\hline mehr Sachverstand & 20 & 8 & 36 \\
\hline
\end{tabular}

Als möglicher Koalitionspartner der SPD boten sich 2007 erneut die Grünen an. Ihr klares Ziel war es, die Große Koalition zu beenden und nach zwölf Jahren parlamentarischer Opposition selbst wieder Regierungsverantwortung zu übernehmen. Vehement kritisierten die Bremer Grünen die angebliche Erfolgsbilanz der Großen Koalition und beklagten unter anderem eine mangelhafte Haushaltssanierung, verschwenderische Projekte, Gefälligkeitsgutachten und unnötige Umzüge der öffentlichen Verwaltung ${ }^{23}$. Nach Auffassung der Grünen hatte die Große Koalition „mit ihrer Arroganz der Macht das Ringen um die besten Lösungen behindert " ${ }^{24}$, und sie brachten damit ein durchaus gewichtiges Argument gegen langjährige Koalitionen vor.

Ihren Wahlkampf stellten die Bündnisgrünen unter das Motto „Die Alternative - Bündnis 90/Die Grünen“ und wählten als zentrale Themen die Bremer Haushaltslage, Bildung und Zukunft von Kindern sowie den Klimaschutz. Mit dieser Schwerpunktsetzung reagierten sie auf aktuelle landes- und bundespolitische Problemlagen. Die grüne Spitzenkandidatin, Karoline Linnert, konnte die Themen zudem glaubhaft vermitteln. Als Vorsitzende des Krankenhaus-Untersuchungsausschusses ${ }^{25}$ hatte die in Politik und Öffentlichkeit weithin anerkannte Fraktionsvorsitzende darüber hinaus in den Monaten vor der Wahl zusätzlich an Renommee gewonnen.

21 Vgl. www.roewekamp.de.

22 Vgl. Forschungsgruppe Wahlen e.V., a.a.O., S. 28.

23 Vgl. hierzu das Wahlprogramm der Grünen „Bremens Alternative“, beschlossen auf der Landesmitgliederversammlung am 11. November 2006, S. 6 f.

24 Ebenda, S. 6.

25 Die genaue Bezeichnung lautete: „Untersuchungsausschuss zur Aufklärung von Schädigungen der kommunalen Krankenhäuser durch Entscheidungen von Geschäftsführern und durch mangelnde Steuerung und Kontrollversagen". 
Ebenso wie die Grünen bekämpfte auch die Bremer FDP die Große Koalition. Angesichts ihrer schwachen Stellung - aktuell war sie nur mit einem Abgeordneten in der Bürgerschaft vertreten und zuvor zweimal an der Fünf-Prozent-Hürde gescheitert - stand jedoch eine Koalition mit der CDU nicht zur Debatte. Ein Wiedereinzug in Fraktionsstärke galt stattdessen schon als ein großer Erfolg, und mit einem verjüngten Team und den Themen Arbeit, Bildung und Sicherheit ${ }^{26}$ versuchten die Bremer Liberalen diesem Ziel näher zu kommen. Neuer Spitzenkandidat war der 40-jährige Ingenieur und Pressesprecher Magnus Bublert, dessen Bekanntheitsgrad in Bremen allerdings eher gering blieb ${ }^{27}$.

Gleichfalls in der Öffentlichkeit kaum bekannt waren die Kandidaten der Linkspartei. Allerdings beflügelte das mit 8,4 Prozent herausragende Abschneiden bei der Bundestagswahl 2005 in Bremen die aus WASG und PDS entstandene Linkspartei und ließ einen Einzug in die Bremer Bürgerschaft als durchaus realistisch erscheinen. Mit ihrem Spitzenkandidaten Peter Erlanson, Diplom-Psychologe und Betriebsrat, zog die Linkspartei in den Wahlkampf und erhielt dabei auch Unterstützung durch ihre prominenten Vertreter der Bundesebene, Oskar Lafontaine und Gregor Gysi. Inhaltlich wandte sich die Linke in einem Elf-Punkte-Aktionsprogramm gegen Sozialabbau und weitere Privatisierungen; sie forderte unter anderem den Ausbau von Ganztagsschulen, mehr Förderung für Kinder mit Migrationshintergrund sowie eine bürgernahe Verwaltung.

Insgesamt verlief der Bremer Wahlkampf eher ruhig und ohne besondere Spannung. Lediglich zum Schluss verschärfte sich der Ton zwischen den beiden großen Parteien im Streit um die frühere RAF-Terroristin Susanne Albrecht, die - wie die Bild-Zeitung berichtet hatte - eine Lehrtätigkeit an einer Bremer Grundschule ausübte. Während die CDU dieses harsch verurteilte, verteidigte die SPD dagegen ihre Einstellung ${ }^{28}$.

\section{Das Wablergebnis}

Der Wahlausgang bestätigte die SPD auch nach 60 Jahren als stärkste Partei im Bundesland Bremen; gleichwohl musste sie - ebenso wie die CDU - deutliche Verluste hinnehmen. Statt der in Umfragen zuletzt prognostizierten 42 Prozent $^{29}$ erzielten die Sozialdemokraten lediglich 36,7 Prozent und büßten damit im Vergleich zur vorherigen Bürgerschaftswahl 5,6 Prozentpunkte ein. Noch schlechter hatte sie bei Landtagswahlen allein 1995 abgeschnitten ${ }^{30}$. Die CDU verlor 4,2 Prozentpunkte und kam nur noch auf 25,6 Prozent, was zugleich ihr

26 Vgl. Arbeit, Bildung, Sicherheit. Wahlprogramm der FDP-Bremen zur Bürgerschaftswahl 2007. Diese Themen fanden sich auch auf ihren Wahlplakaten: „Arbeitsplätze statt Subventionen“, „Kinder fördern statt verwalten“ oder „Sicherheit statt schönreden“ war dort zu lesen.

27 Eckhard Stengel zufolge hat die FDP „einen Spitzenkandidaten, der in Bremen etwa so bekannt ist wie der rumänische Staatspräsident". Vgl. Eckhard Stengel, Partner-Poker an der Weser, in: Frankfurter Rundschau (FR) vom 9. Mai 2007, S. 3.

28 Vgl. Ralf Wiegand, Streit über Ex-Terroristin. Susanne Albrecht wird zum Wahlkampfthema in Bremen, in: Süddeutsche Zeitung (SZ) vom 4. Mai 2007, S. 6; Bernd Schneider, Streit um Susanne Albrecht wird schärfer, in: WK vom 5. Mai 2007, S. 11.

29 Nach einer Umfrage von Infratest dimap vom April 2007 kamen die Parteien auf folgende Werte: SPD: 42, CDU: 26, Bündnisgrüne: 14,5, Linke: 5,5 und FDP: 5 Prozent. Vgl. www.infratestdimap.de (LänderTREND Bremen April 2007).

30 Bei der Bürgerschaftswahl 1995 erzielte die SPD lediglich 33,9 Prozent. Vgl. auch Reinhold Roth, a.a.O. 
zweitschlechtestes Ergebnis in den letzten 20 Jahren darstellte. Demgegenüber konnten die kleinen Parteien große Erfolge verbuchen. Die Grünen gewannen 3,6 Prozentpunkte hinzu und erzielten mit 16,4 Prozent ihr bestes Ergebnis nicht nur in Bremen, sondern bei Landtagswahlen überhaupt. Die Linkspartei schaffte mit 8,4 Prozent erstmalig den Sprung in ein westdeutsches Landesparlament und wiederholte damit exakt ihr Wahlergebnis bei der Bundestagswahl 2005 im Land Bremen. Die FDP verbesserte sich um 1,7 Prozentpunkte und erreichte nun 5,9 Prozent. Von den 83 Sitzen der Bremer Bürgerschaft entfielen damit 33 auf die SPD und 23 auf die CDU; die Grünen erhielten 14, die Linke sieben und die FDP fünf Mandate. Aufgrund der getrennten Fünf-Prozent-Klausel für die Wahlbereiche Bremen und Bremerhaven zog auch die DVU mit einem Abgeordneten erneut in die Bürgerschaft ein, da sie 5,3 Prozent in Bremerhaven erhalten hatte (vgl. Tabelle 2) ${ }^{31}$. Mit nunmehr fünf in der Bürgerschaft in Fraktionsstärke vertretenen Parteien kann auch in diesem Bundesland eine Ausdehnung auf ein Fünfparteiensystem konstatiert werden.

\begin{tabular}{|c|c|c|c|c|c|c|c|c|}
\hline \multicolumn{9}{|c|}{$\begin{aligned} & \text { Tabelle 2: } \text { Ergebnis der Bremer Bürgerschaftswabl vom 13. Mai } 2007 \\
& \text { (mit Vergleichsdaten zu früheren Wablen) }\end{aligned}$} \\
\hline & \multicolumn{3}{|c|}{$\begin{array}{l}\text { Bürgerschaftswahl } \\
13.5 .2007\end{array}$} & \multicolumn{3}{|c|}{$\begin{array}{c}\text { Bürgerschaftswahl } \\
25.5 .2003\end{array}$} & \multicolumn{2}{|c|}{$\begin{array}{l}\text { Bundestagswahl } \\
\text { 18.9.2005 }\end{array}$} \\
\hline & absolut & Prozent & Sitze & absolut & Prozent & Sitze & absolut & Prozent \\
\hline Wahlberechtigte & 486.073 & & & 481.743 & & & 486.475 & \\
\hline Wähler & 279.855 & 57,6 & & 295.391 & 61,3 & & 367.282 & 75,5 \\
\hline $\begin{array}{l}\text { Gültige Stimmen } \\
\text { davon: }\end{array}$ & 276.022 & & 83 & 291.766 & & 83 & 361.921 & \\
\hline SPD & 101.417 & 36,7 & 33 & 123.480 & 42,3 & 40 & 155.366 & 42,9 \\
\hline $\mathrm{CDU}$ & 70.832 & 25,6 & 23 & 86.819 & 29,8 & 29 & 82.389 & 22,7 \\
\hline Grüne & 45.524 & 16,4 & 14 & 37.350 & 12,8 & 12 & 51.600 & 14,2 \\
\hline FDP & 16.497 & 5,9 & 5 & 12.294 & 4,2 & 1 & 29.329 & 8,1 \\
\hline Die Linke & 23.296 & 8,4 & 7 & 4.885 & 1,7 & & 30.570 & 8,4 \\
\hline DVU & 7.566 & 2,7 & 1 & 6.642 & 2,3 & 1 & & \\
\hline BIW & 2.216 & 0,8 & & & & & & \\
\hline REP & 1.430 & 0,5 & & & & & & \\
\hline Deutschland & 338 & 0,1 & & & & & & \\
\hline Die Konservativen & 4.465 & 1,6 & & & & & & \\
\hline Die Weißen & 171 & 0,06 & & & & & & \\
\hline Die Frauen & 1.318 & 0,4 & & 1.098 & 0,4 & & 1.452 & 0,4 \\
\hline PBC & 952 & 0,3 & & 1.009 & 0,4 & & 1.083 & 0,3 \\
\hline Sonstige & & & & 18.189 & $6,1^{*}$ & & 10.122 & 3,0 \\
\hline \multicolumn{9}{|c|}{$\begin{array}{l}\text { BIW = Bürger in Wut; Deutschland = Ab jetzt ... Bündnis für Deutschland, Partei für Demokratie } \\
\text { durch Volksabstimmung; Die Weißen = Die Weißen. Demokratische Alternative; Die Frauen = Feminis- } \\
\text { tische Partei Die Frauen; PBC = Partei Bibeltreuer Christen. } \\
\text { * Darunter 4,4 Prozent }(=12.876 \text { Stimmen) für die Partei Rechtsstaatlicher Offensive (Schill-Partei). } \\
\text { Quelle: Statistisches Landesamt Bremen, Wahl zur Bremischen Bürgerschaft im Lande Bremen am } \\
\text { 13. Mai 2007. Endgültige Ergebnisse. }\end{array}$} \\
\hline
\end{tabular}

31 Die nur im Wahlbereich Bremerhaven angetretene Wählervereinigung „Bürger in Wut“ erzielte 4,99 Prozent; damit fehlte ihr exakt eine Stimme für einen Sitz im Parlament. Vgl. Statistisches Landesamt Bremen, Statistische Mitteilungen, Heft 110/2007, S. 12. Im Frühjahr 2008 stellte der Bremer Staatsgerichtshof nach Einspruch der BIW in drei von 95 Wahlbezirken in Bremerhaven Wahlfehler fest und ordnete für einen Wahlbezirk eine Wahlwiederholung an. Vgl. Gericht ordnet Teil-Neuwahl an, in: Das Parlament vom 26. Mai 2008. 
Die Mehrheit der Abgeordneten in der Bremischen Bürgerschaft ist nach wie vor männlich; zudem ging der Frauenanteil erstmals seit 1991 wieder zurück. Insgesamt zogen 32 Frauen in das Parlament ein, was einem Anteil von 38,5 Prozent entspricht (2003: 41 Prozent). Den höchsten Frauenanteil können nach wie vor die Grünen mit 50 Prozent vorweisen; es folgen die SPD mit 45, die Linke mit 43 und die CDU mit 30 Prozent. Unter den Abgeordneten von FDP und DVU findet sich keine Frau (vgl. Tabelle 3). Auffällig ist eine gleichfalls rückläufige Tendenz des Frauenanteils auf den Listen von SPD, CDU und Grünen. Möglicherweise zeigen sich hier gewisse Ermüdungserscheinungen der Parteien in ihrem Bestreben, gleiche politische Teilhabechancen für Frauen zu gewährleisten.

\begin{tabular}{|l|c|c|c|c|c|c|}
\hline Tabelle 3: Kandidaturen und Gewählte nach Geschlecht bei der Bremer Bürgerschaftswahl \\
vom 13. Mai 2007
\end{tabular}

Eine breite Mobilisierung der Wählerschaft war den Parteien in ihrem spannungsarmen Wahlkampf erneut nicht gelungen. Die zuletzt ohnehin schon niedrige Wahlbeteiligung (2003: 61,3 Prozent) verringerte sich 2007 weiter auf magere 57,6 Prozent und erreichte damit im Land Bremen ihren historischen Tiefstand ${ }^{32}$.

32 Vgl. Statistisches Landesamt Bremen, Statistisches Jahrbuch 2004, Bremen (Dezember) 2004, S. 37. 
Betrachtet man die Wahlbeteiligungsquoten im Einzelnen, dann zeigen sich nicht nur erhebliche Unterschiede zwischen den beiden Wahlbereichen (Stadt Bremen: 58,5 Prozent; Bremerhaven: 52,8 Prozent), sondern auch zwischen den verschiedenen Stadtquartieren. In traditionellen Arbeiterwohnvierteln in der Stadt Bremen, wie zum Beispiel Gröpelingen (47,1 Prozent), blieb die Wahlbeteiligung unter 50 Prozent, während im gutbürgerlichen Schwachhausen durchweg über 70 Prozent erzielt wurden. Entsprechendes gilt auch für die unterschiedlich strukturierten Stadtteile Bremerhavens ${ }^{33}$. Damit belegen diese empirischen Befunde erneut den bekannten Zusammenhang zwischen der individuellen Wahlbeteiligung und den sozialräumlichen Strukturen der Stadtteile. Bemerkenswert ist allerdings, dass in „Gebieten mit gewachsener sozialer Benachteiligung, den sogenannten prekären Lebenslagen, ... der Rückgang diesmal am höchsten “34 war.

Unterschiede zwischen den Wahlbereichen zeigen sich auch hinsichtlich der Stimmabgabe. Mit Ausnahme von FDP und DVU erzielten die Bürgerschaftsparteien in der Stadt Bremen bessere Ergebnisse als in Bremerhaven. So konnte die SPD in Bremen 37,1 Prozent für sich verbuchen, in Bremerhaven dagegen nur 34,9 Prozent. Bei der CDU fielen die Differenzen mit 25,7 Prozent (Bremen) zu 25,1 Prozent (Bremerhaven) diesmal eher gering aus. Die Grünen dagegen waren in Bremen mit 17,3 Prozent deutlich stärker als in Bremerhaven mit 11,9 Prozent. Und auch die Linkspartei schnitt in Bremen mit 8,7 Prozent erkennbar besser $\mathrm{ab}$ als in Bremerhaven mit 6,9 Prozent. Im Gegensatz dazu erhielt die FDP in Bremerhaven deutlich mehr Stimmen (8,7 Prozent) als in Bremen (5,4 Prozent). Die DVU schließlich scheiterte in Bremen erneut an der Fünf-Prozent-Hürde (2,2 Prozent), während sie in Bremerhaven auf 5,3 Prozent kam ${ }^{35}$.

Im Vergleich zur Bürgerschaftswahl 2003 musste die SPD insbesondere in den innerstädtischen Bereichen gravierende Stimmenverluste zwischen 11,0 und 12,5 Prozentpunkten hinnehmen. In ihren Hochburgen dagegen, den älteren Arbeitervierteln und Großsiedlungen, konnte sie erneut ihre besten Ergebnisse erzielen, und die Verluste fielen hier mit etwa 3,0 Prozentpunkten unterdurchschnittlich aus ${ }^{36}$. Mit nur leichten Verlusten konnte auch die CDU ihre traditionellen Hochburgen, die bürgerlichen Wohnviertel mit eher konservativem Milieu, als stärkste Partei verteidigen. Die geringsten Stimmenanteile erhielten die Christdemokraten in den Ortsteilen der City und des Cityrandbereiches. Daneben gab es aber auch in den Hochburgen der SPD empfindliche Stimmenverluste für die $\mathrm{CDU}^{37}$. Demgegenüber konnten die Grünen ihre Hochburgen um den Stadtkern Bremens weiter ausbauen und wurden hier mit durchschnittlich 34 Prozent die erste politische $\mathrm{Kraft}^{38}$. Für die FDP sind die größten Erfolge in den traditionell bürgerlichen Vierteln zu verzeichnen. Die Linke hat ihre Hochburgen in den zentrumsnahen Ortsteilen sowie in den Großsiedlungen und Arbeitervierteln, in den bürgerlichen Wohnvierteln dagegen ist sie kaum verankert ${ }^{39}$. Die DVU erreichte in Bremerhaven diesmal keine zweistelligen Er-

33 Vgl. Statistisches Landesamt Bremen, Statistische Mitteilungen, Heft 110/2007, S. 10.

34 Ebenda, S. 13.

35 Vgl. Statistisches Landesamt Bremen, Wahl zur Bremischen Bürgerschaft (Landtag) im Lande Bremen am 13. Mai 2007, Endgültige Ergebnisse (Online-Dokument unter www.statistik.bremen.de).

36 Vgl. Statistisches Landesamt Bremen, Statistische Mitteilungen, Heft 110/2007, S. 13.

37 Vgl. ebenda, S. $13 \mathrm{f}$.

38 Vgl. ebenda, S. 12.

39 Vgl. ebenda. 
gebnisse mehr; in der Stadt Bremen kam sie nur in einem Wahlbezirk auf über fünf Prozent $^{40}$.

Die Aufschlüsselung des Wahlergebnisses nach Berufsgruppen zeigt im Vergleich zu 2003 einige Veränderungen: Die SPD konnte allein bei den Arbeitern mit 45 Prozent ihr Resultat halten; von den gewerkschaftlich organisierten Arbeitern erhielt sie sogar 52 Prozent und damit drei Prozentpunkte mehr als noch 2003. Demgegenüber sackte die CDU in der Gunst der Arbeiterschaft weiter ab und überzeugte nur noch jeden fünften Wähler (-9). Deutliche Gewinne erzielte dagegen die Linke unter den Arbeitern; mit 12 Prozent wurde sie hier drittstärkste Kraft $(+10)^{41}$.

Bei den Angestellten verlor die SPD sieben Punkte und erreichte nur noch 38 Prozent, während die CDU auf 26 Prozent kam (-3). Die Grünen dagegen gewannen in dieser Berufsgruppe leicht hinzu und erzielten 18 Prozent. Deutlich höher fielen ihre Gewinne unter den Beamten aus, wo sie sich auf beachtliche 22 Prozent steigern konnten (+8). Demgegenüber rutschte die SPD bei den Staatsdienern auf 32 Prozent ab $(-10)$, während sich die CDU hier entgegen dem Trend leicht verbessern konnte und auf 29 Prozent kam (+2). Ihr bestes Ergebnis erhielt die CDU mit 37 Prozent jedoch bei den Selbständigen, gleichwohl mit deutlichem Verlust gegenüber 2003 (-7). Auch die SPD musste hier Abzüge verbuchen und erreichte nur noch 21 Prozent (-7). Im Vergleich dazu schnitt die FDP in dieser Gruppe mit neun Prozent klar überdurchschnittlich $\mathrm{ab}^{42}$.

Hinsichtlich des Einflusses der Konfession auf die Wahlentscheidung zeigten sich die bekannten Effekte: Die in Bremen nur kleine Gruppe von Katholiken stimmte zu 39 Prozent für die CDU und nur zu 27 Prozent für die SPD. Protestanten dagegen bevorzugten mit 40 Prozent die SPD (CDU: 27 Prozent) ${ }^{43}$.

Bei der Wahlentscheidung nach Bildungsgruppen ${ }^{44}$ lag die SPD - mit Ausnahme der Hochschulabsolventen - überall vorn; ihr bestes Ergebnis erreichte sie bei Wählern mit Hauptschulabschluss (48 Prozent). Demgegenüber erhielt die CDU den höchsten Zuspruch von Wählern mit Mittlerer Reife (28 Prozent). Deutlich über dem Durchschnitt lag das Votum der Wähler mit Hochschulreife sowie Hochschulabschluss für die Grünen (22 beziehungsweise 33 Prozent). Im Unterschied dazu erzielte die Linke in allen Bildungsgruppen annähernd ihr Landesergebnis. Entsprechendes galt auch für die FDP, die gegenüber 2003 zudem in allen Bildungsgruppen hinzugewinnen konnte. Den Schwerpunkt der DVU bildeten in Bremerhaven Wähler mit Hauptschulabschluss beziehungsweise Mittlerer Reife.

Auch bei dieser Wahl zeigten sich geschlechtsspezifische Unterschiede, allerdings fielen sie etwas schwächer aus als vor vier Jahren ${ }^{45}$. So votierten Frauen erneut stärker für die SPD als Männer (40,2 beziehungsweise 34,9 Prozent), und auch die Grünen wurden leicht von

40 Vgl. Statistisches Landesamt Bremen, Wahl zur Bremischen Bürgerschaft 2007; Stadt Bremen Orts- und Stadtteilergebnisse; Stadt Bremerhaven - Orts- und Stadtteilergebnisse (unter www. statistik.bremen.de).

41 Vgl. Forschungsgruppe Wahlen, a.a.O., S. 43 f.

42 Vgl. ebenda.

43 Vgl. ebenda, S. 46.

44 Vgl. ebenda, S. $48 \mathrm{f}$.

45 Vgl. Jürgen Wayand, Ergebnisse der Bürgerschaftswahl 2003 im Lande Bremen nach Altersgruppen und Geschlecht, in: Statistische Monatsberichte Bremen, Heft 9/10, Bremen 2003, S. $200 \mathrm{ff}$. 
Frauen bevorzugt (17,2 beziehungsweise 15,3 Prozent). Für die CDU ergab sich diesmal ein annähernd gleiches Stimmverhalten der Geschlechter (Männer: 24,5, Frauen: 24,2 Prozent). Deutlich attraktiver für Männer war dagegen die Linke (10,8 beziehungsweise 6,7 Prozent), aber auch FDP und DVU verzeichneten mehr Männer- als Frauenstimmen ${ }^{46}$.

In allen Altersgruppen war die SPD führend. Den geringsten Zuspruch bekam sie erneut von den 18- bis unter 25-Jährigen, den höchsten von den über 60-Jährigen (40,4 Prozent). In dieser Altersgruppe hatte auch die CDU ihren größten Erfolg (33,5 Prozent); in den übrigen Gruppen blieb sie dagegen stets unter 20 Prozent ${ }^{47}$. Im Vergleich zu 2003 verloren beide Parteien durchweg in allen Altersgruppen, wobei die höchsten Einbußen für die 45- bis unter 60-Jährigen zu verzeichnen waren (SPD: -6,4, CDU: -5,3) ${ }^{48}$. Die Grünen konnten mit 27,3 Prozent insbesondere bei den 18- bis unter 25-Jährigen punkten $(+8,6)$, erreichten aber auch in den anderen Altersklassen - mit Ausnahme der Wähler ab 60 Jahren - überdurchschnittliche Anteile. Für FDP und Linke ergab die altersspezifische Verteilung der Stimmenanteile ein weitgehend ausgeglichenes Bild; allein bei den 45- bis unter 60-Jährigen kam die Linke auf ein zweistelliges Ergebnis (11,7 Prozent ${ }^{49}$.

Betrachtet man abschließend die vermuteten Wählerströme, dann hat die SPD vor allem an die Nichtwähler (-7.000), die Linke (-6.000) und die Grünen (-5.000) Stimmen abgeben müssen. Auch die CDU litt erheblich unter der niedrigen Wahlbeteiligung (-8.000). Daneben verstarben mehr Unionswähler, als sie unter den Erstwählern neu hinzugewinnen konnte, was mit einem Saldo von 6.000 Stimmen zu Buche schlug. Die Grünen konnten 5.000 ehemalige SPD-Wähler für sich gewinnen, verloren aber an die Linkspartei 3.000 und an die FDP 1.000 Wähler. Zur Linkspartei wechselten neben den 3.000 ehemaligen Wählern der Grünen auch 6.000 von der SPD und 1.000 von der CDU. Die FDP erhielt von der CDU 2.000 Wähler, von der SPD und den Grünen jeweils 1.000. An die Gruppe der Nichtwähler verlor sie 1.000 Stimmen $^{50}$.

\section{Regierungs- und Oppositionsbildung}

Der Ausgang der Bürgerschaftswahl eröffnete der SPD die Option für ein rot-schwarzes wie für ein rot-grünes Regierungsbündnis. Bundespolitische Auswirkungen waren allerdings nur bei einer Koalition mit den Grünen zu erwarten, denn in diesem Falle würden die beiden Regierungsparteien im Bundesrat ihre Zweidrittelmehrheit verlieren und könnten zukünftig keine Verfassungsänderungen mehr allein beschließen. Auch wenn entsprechende Überlegungen durchaus eine Rolle gespielt haben, gab es offiziell von Seiten der BundesSPD keine Einflussnahme. So sagte der Parteivorsitzende Kurt Beck, er habe „keine Präferenz" für ein Regierungsbündnis in Bremen; vielmehr gehe es darum, „ein Optimum an sozialdemokratischer Politik“ zu verwirklichen ${ }^{51}$. Demgegenüber sah die Parteivorsitzende

46 Vgl. Horst Lange, Ergebnisse der Bürgerschaftswahl 2007 im Lande Bremen nach Altersgruppen und Geschlecht, in: Statistisches Landesamt Bremen, Statistische Hefte 1/2007, S. 10 ff.

47 Vgl. ebenda.

48 Vgl. Jürgen Wayand, a.a.O., S. $200 \mathrm{ff}$.

49 Vgl. Horst Lange, a.a.O., S. $10 \mathrm{ff}$.

50 Vgl. Infratest dimap Wahlreport, Bürgerschaftswahl Bremen 2007, S. 31 ff.

51 Zitiert nach: Bremen-Wahl: SPD grübelt - Lafontaine jubelt, in: Spiegel Online vom 14. Mai 2007 (www.spiegel.de/politik/deutschland/0,1518,druck-482688,00.html). Für eine Koalition 
der Grünen, Claudia Roth, angesichts des Erfolgs ihrer Partei einen „ganz klaren Auftrag für die Übernahme von Regierungsverantwortung" ${ }^{2}$. Seitens der CDU im Bund wies Generalsekretär Ronald Pofalla den Sozialdemokraten die Schuld am schlechten Abschneiden der Großen Koalition zu: „Sie haben keine klare Aussage zur Fortsetzung der erfolgreichen Regierung in Bremen gemacht." 53

Die Bremer SPD-Spitze nahm zunächst Sondierungsgespräche mit den Grünen wie auch der CDU auf, die im letzteren Fall jedoch ein schnelles Ende fanden. Bereits eine Woche nach der Wahl erteilte die CDU den Sozialdemokraten eine Absage und entschied sich für die Oppositionsrolle. Der CDU-Landesvorsitzende Bernd Neumann und Bürgermeister Thomas Röwekamp bezeichneten die Sondierungsgespräche als „unaufrichtig gegenüber der CDU ... , da sich die SPD bereits in den letzten Wochen deutlich von der großen Koalition verabschiedet und auf Rot-Grün nach der Wahl gesetzt ${ }^{\text {"54 }}$ habe.

Nachdem sich die Parteiversammlungen von SPD und Grünen jeweils nahezu einstimmig für die Aufnahme von Koalitionsverhandlungen ausgesprochen hatten, gingen die Verhandlungen zügig voran. In mehreren Gesprächsrunden wurden zunächst inhaltliche Fragen geklärt, wobei unter anderem Finanzen, Wirtschaft und Häfen sowie Baupolitik im Mittelpunkt standen ${ }^{55}$. Strittige Themen waren insbesondere die geplante Vertiefung der Außenweser sowie der Bau eines neuen Kohlekraftwerkes. Während die Grünen der Weservertiefung unter der Auflage zustimmten, dass weitgehende ökologische Ausgleichsmaßnahmen vorgenommen werden, wurde hinsichtlich des Kohlekraftwerks vereinbart, zunächst ein ergebnisoffenes Prüf- und Moderationsverfahren einzuleiten.

Der schließlich in einem guten Klima ausgehandelte Koalitionsvertrag setzte drei Schwerpunkte: die Stärkung der Wirtschaftskraft des Landes, den Ausbau des sozialen Zusammenhalts und der Chancengleichheit sowie die Sicherung der Selbständigkeit Bremens ${ }^{56}$. Die Parteitage von SPD und Grünen stimmten dem Koalitionsvertrag jeweils mit großer Mehrheit zu, und am 27. Juni 2007 fand die Unterzeichnung statt.

Zwei Tage später wurden die von der Bremischen Bürgerschaft gewählten Mitglieder des Senats vereidigt. Von den nach wie vor sieben Senatsposten gingen fünf an die SPD und zwei an die Grünen (vgl. Tabelle 4). Alter wie neuer Präsident des Senats sowie Kultursenator ist Jens Böhrnsen. Für die SPD wechselte Willi Lemke vom Bildungs- in das Innen- und Sportressort; Ingelore Rosenkötter bleibt zuständig für Arbeit, Frauen, Gesundheit, Jugend und Soziales. Neue Senatorin für Bildung und Wissenschaft ist die frühere niedersächsische Kultusministerin Renate Jürgens-Pieper, und gleichfalls neu im Senat ist der ehemalige Staatssekretär im Bundesverkehrsministerium Ralf Nagel, der das Ressort Wirtschaft und

mit den schon zweimal abgewiesenen Grünen sprach seitens der Bremer SPD aber auch die Überlegung, nicht den Boden für ein eventuelles schwarz-grünes Bündnis bei der Bürgerschaftswahl 2011 bereiten zu wollen. So äußerte sich der SPD-Landesgeschäftsführer Roland Pahl im Gespräch mit der Verfasserin (B.H.).

52 Ebenda.

53 Ebenda.

54 Zitiert nach: CDU-Absage: Bremer SPD will rot-grüne Koalition, in: Spiegel Online vom 20. Mai 2007 (www.spiegel.de/politik/deutschland/0,1518,druck-483777,00.html).

55 Vgl. Wigbert Gerling, Fördergeld für Betriebe nur noch Leihgabe? Rot-grüne Ansätze zur Akzentverschiebung in der Wirtschaftsförderung, in: WK vom 1. Juni 2007, S. 9.

56 Vgl. Vereinbarung zur Zusammenarbeit in einer Regierungskoalition für die 17. Wahlperiode der Bremischen Bürgerschaft 2007-2011. 
Tabelle 4: Der Senat des Landes Bremen nach den Wablen vom 13. Mai 2007

Präsident des Senats und Kultursenator

Senatorin für Finanzen

Senator für Inneres und Sport

Senatorin für Arbeit, Frauen, Gesundheit, Jugend und Soziales

Senatorin für Bildung und Wissenschaft

Senator für Umwelt, Bau, Verkehr und Europa

Senator für Wirtschaft und Häfen, Justiz und Verfassung
Jens Böhrnsen (SPD)

Karoline Linnert (Grüne)

Willi Lemke (SPD)

Ingelore Rosenkötter (SPD)

Renate Jürgens-Pieper (SPD)

Dr. Reinhard Loske (Grüne)

Ralf Nagel (SPD)

Häfen sowie Justiz übernimmt ${ }^{57}$. Für die Grünen gehören der Landesregierung Karoline Linnert als Finanzsenatorin und Bürgermeisterin an sowie der bisherige nordrhein-westfälische Bundestagsabgeordnete Reinhard Loske als Senator für Umwelt, Bau, Verkehr und Europa. Mit drei Senatorinnen (zuvor eine) ist die neue Bremer Regierung nunmehr deutlich „weiblicher" geworden.

Auf der Parlamentsebene sieht sich das Regierungsbündnis einer zahlenmäßig starken Opposition gegenüber. Thomas Röwekamp als neuer Fraktionschef der CDU freut sich „auf die hoffentlich kurze Zeit in der Opposition"58. Die FDP-Fraktion hat Uwe Woltemath zu ihrem Fraktionsvorsitzenden gewählt, und die Linkspartei wird in der Bürgerschaft von Monique Troedel und Peter Erlanson geführt. Zum Präsidenten der Bürgerschaft wurde erneut Christian Weber (SPD) gewählt.

In seiner ersten Regierungserklärung vor der Bürgerschaft erläuterte Böhrnsen die wesentlichen Eckpunkte der Regierungsarbeit und betonte das neue Selbstverständnis in der Koalition: „Nicht Ressortdenken und Fingerhakeln um einzelne Themen bestimmen die zukünftige Arbeit, sondern das Selbstverständnis einer gemeinsamen Verantwortung für alle Politikbereiche. Rot-grün steht für eine geschlossene Mannschaftsleistung. "59

\section{Zusammenfassung}

(1) Die Beteiligung an der Wahl zur Bremischen Bürgerschaft am 13. Mai 2007 erreichte mit 57,6 Prozent ihren historischen Tiefstand. In Bremerhaven machte nahezu nur jeder zweite Wahlberechtigte von seinem Stimmrecht Gebrauch.

(2) Die SPD blieb stärkste Partei im Stadtstaat Bremen, musste aber erneut deutliche Verluste hinnehmen. Mit 36,7 Prozent kam sie auf ihr zweitschlechtestes Ergebnis seit 1947. Im Vergleich zur CDU konnte sie jedoch von der positiven Beurteilung ihres Spitzenkandidaten, erhöhtem Zuspruch bei der Sachkompetenz sowie der guten Bewertung ihrer Senatsarbeit profitieren. Inhaltlich hatte sie mit den Themen Arbeit, Bildung und sozialer Zusammenhalt die überzeugendere Wahlkampfstrategie verfolgt.

57 In dieses Ressort sollte ursprünglich der bisherige parteilose Finanzsenator Ulrich Nußbaum wechseln. Dieser hatte jedoch überraschend abgesagt, da er massiv bedrängt worden sei, in die SPD einzutreten. Dieses lehnte er ab und sah für seine Arbeit im Senat nicht mehr den erforderlichen Rückhalt. Vgl. Jürgen Hinrichs, Jens Böhrnsen gelingt ein schneller Wurf. Ralf Nagel wird neuer Wirtschaftssenator in Bremen, in: WK vom 22. Juni 2007, S. 1.

58 So Röwekamp auf seiner Homepage (www.roewekamp.de/arbeit/index.php).

59 Regierungserklärung von Bürgermeister Jens Böhrnsen am 11. Juli 2007 vor der Bremischen Bürgerschaft (Online-Dokument unter www.jens-boehrnsen.de). 
(3) Die CDU konnte von ihrer Regierungsbeteiligung nicht profitieren und verlor auf zentralen Politikfeldern ihren bisherigen Kompetenzvorsprung. Ein eher blasser Spitzenkandidat sowie ein inhaltlich verfehlter Wahlkampf ließen sie auf 25,6 Prozent abrutschen. Nach zwölf Jahren im Senat muss sich die Bremer CDU nunmehr mit der Oppositionsrolle begnügen.

(4) Die Grünen erzielten 16,4 Prozent und damit ihr bestes Ergebnis bei Landtagswahlen überhaupt. Insbesondere unter den jüngeren Wählern kamen sie auf überdurchschnittliche Anteile. Mit der Regierungsbeteiligung im neuen rot-grünen Senat haben die Bremer Grünen ihr Wahlkampfziel erreicht.

(5) Die Linkspartei schaffte mit 8,4 Prozent auf Anhieb den Sprung in die Bürgerschaft und ist damit zum ersten Mal in einem westdeutschen Landesparlament vertreten.

(6) Die FDP bekam 5,9 Prozent und konnte erstmals seit 1995 wieder in Fraktionsstärke in die Bürgerschaft einziehen. Deutlich erfolgreicher als in Bremen war sie in Bremerhaven mit 8,7 Prozent.

(7) Die parlamentarische Vertretung von Frauen ist erstmals seit 1991 wieder rückläufig. Nach zuletzt 41 Prozent beträgt der Frauenanteil in der Bremischen Bürgerschaft nur noch 38,5 Prozent. Im Senat hat er sich hingegen verdreifacht.

(8) Mit dem Schwenk zu den Grünen beendete die SPD die langjährige Große Koalition, auch wenn es in der Bevölkerung keine ausgeprägte Wechselstimmung gab.

(9) Insgesamt bestätigt der Wahlausgang, dass Große Koalitionen die kleinen Parteien stärken. Mit dem Einzug von Linkspartei und FDP jeweils in Fraktionsstärke in die Bürgerschaft hat sich das bisherige stabile Bremer Dreiparteiensystem zu einem Fünfparteiensystem erweitert.

\title{
Die niedersächsische Landtagswahl vom 27. Januar 2008: Popularität, Wahlstrategie und Oppositionsschwäche sichern Ministerpräsident Christian Wulff die schwarz-gelbe Mehrheit
}

\author{
Ralf Tils und Thomas Saretzki
}

\section{Die Ausgangslage}

Die Niedersachsen sind interessante Wähler. Einerseits bescheren sie ihren Landesregierungen regelmäßig längere Amtsperioden, so dass sich in der niedersächsischen Geschichte ausgedehnte Zeiträume der Dominanz entweder der Konservativen oder der Sozialdemokraten beobachten lassen. So stellte die SPD von 1947 bis zu Anfang der siebziger Jahre mit den Ministerpräsidenten Hinrich Wilhelm Kopf, Georg Diederichs und Alfred Kubel fast ${ }^{1}$ durch-

1 Diese sozialdemokratische Dominanz wurde nur 1955 bis 1957 durch eine konservative Landesregierung mit Heinrich Hellwege von der Deutschen Partei (DP) an der Spitze unterbrochen, vgl. dazu Helmut Beyer / Klaus Müller, Der Niedersächsische Landtag in den fünfziger Jahren. Voraussetzungen, Ablauf, Ergebnisse und Folgen der Landtagswahl 1955, Düsseldorf 1988. 